\title{
Explorability of Noisy Scalar Fields
}

\author{
Wencen $\mathrm{Wu}$ and Fumin Zhang
}

\begin{abstract}
We consider mobile sensing agents taking measurements along their trajectories and navigating in a noisy scalar field. Not knowing the field value at its location, each agent decides its motion based on sensor measurements. When an agent changes its position, both the sensor measurement and the field value at its location change. If the change of the field value is not consistent with the change of the measurement, we say the agent performs a false-walk. We establish the notion of local explorability for the noisy scalar field to analyze the tendency that such field will induce false-walks for a sensing agent. We show that, a field being locally explorable is the sufficient and necessary condition for the probability of inducing a false-walk to be less than a certain threshold. We then connect explorability with the noise reduction capability using multiple sensing agents, and compute the minimum number of agents necessary to ensure explorability.
\end{abstract}

\section{INTRODUCTION}

Exploration of an unknown scalar field is one of the fundamental problems in mobile robotics. Various types of exploration missions are investigated in literature, such as climbing or descending gradients [1]-[4], tracking level sets [5]-[7], cooperative path following [8] and monitoring environmental boundaries [9], [10]. To efficiently and successfully explore an unknown field, some works deal with the development and improvement of the algorithms using one agent [11]-[15], while recent attentions have been paid to using multiple agents to perform cooperative exploration [6], [16], [17] due to its advantages over individual exploration in certain contexts. The problem of exploring unknown fields is also related to map making [18], [19].

Most existing works concern the design of exploring strategies. In this paper, we look into the exploration problem from the analytic point of view, that is, whether a field is difficult to be explored or not. To address this problem, we first propose a concept of "false-walk" performed by a sensing agent moving in a field and taking measurements of the field. A false-walk happens when the sensor reading of an agent increases(decreases) while the mean field value decreases(increases). Then we introduce the definition of "local explorability" of a field, which gives an indicator on how easy a false-walk can happen. We relate the explorable probability with the noise properties of a field, which provides a way to check the explorability if the field is known.

Based on the definition of explorable fields, we analyze an algorithm that attempts to direct an agent to decrease its

The research work is supported by ONR grants N00014-08-1-1007, N00014-09-1-1074, and N00014-10-10712 (YIP), and NSF grants ECCS0841195 (CAREER), CNS-0931576, and ECCS-1056253. Wencen Wu and Fumin Zhang are with the School of Electrical and Computer Engineering, Georgia Institute of Technology, Atlanta, GA, 30332, USA wwencen3, fumin@gatech.edu measured field. We prove that, if a field is locally explorable, then under certain extra conditions, the agent moves in the correct direction with probability larger than $\frac{1}{2}$.

In typical scenarios, multiple agents that are collaborating are expected to perform better than a single agent in terms of adaptiveness and efficiency [20], [21]. And, as the number of agents increases, the noise reduction capability increases due to cooperative filtering algorithms performed [6], [7], [22], [23]. However, in practice, we can not deploy infinite number of agents due to the increasing cost and complexity in control, communications and computations. Therefore, given a cooperative filtering algorithm, we need to find out how many agents are necessary to explore a field. To address this problem in the context of explorability, we look into an example introduced in [6], in which a cooperative Kalman filter is computed by a formation formed by $N$ agents to provide estimated field values at the formation center. The trace of the error covariance matrix of the cooperative Kalman filter provides a measure on the noise reduction capabilities with $N$ agents [6]. Therefore, by relating the error covariance matrix to the explorable probability of the field, we can compute the minimum number of agents necessary to achieve a certain explorable probability.

The rest of the paper is organized as follows. In section II, we introduce the definition of local explorability and discuss the properties of explorable fields. In section III, we analyze an algorithm that is to direct an agent to decrease its measured field value in explorable fields. In section IV, we discuss the cooperative filtering and explorability. In section $\mathrm{V}$, simulation results are provided. Concluding remarks are presented in section VII.

\section{LOCAL EXPLORABILITY}

In this section, we introduce the definitions of falsewalk and local explorability and discuss the properties of explorable fields.

\section{A. Definitions}

Consider a noisy scalar field $Y(\mathbf{x}): \mathbb{R}^{n} \rightarrow \mathbb{R}$, where $\mathbf{x} \in$ $\mathbb{R}^{n}$ is a position in the field. Let $F(\mathbf{x})$ be the mean field $F(\mathbf{x})=\mu(Y(\mathbf{x}))$. Suppose $F(\mathbf{x})$ is smooth. Define the noise of the field to be the difference between $Y(\mathbf{x})$ and $F(\mathbf{x})$, that is $W(\mathbf{x})=Y(\mathbf{x})-F(\mathbf{x})$. For $Y(\mathbf{x})$ and $W(\mathbf{x})$, we use upper cases to denote random variables and lower cases to denote values of random variables.

Suppose a sensing agent is moving in the field while taking measurements of the field discretely. We have the following assumptions throughout the paper: 
1. Each sensing agent takes one measurement of the field value at each time instant $t_{k}$.

2. The field noise is zero-mean and independent, which gives

$$
\begin{aligned}
\mathrm{E}\left(W\left(\mathbf{x}_{k}\right)\right) & =0, \\
\mathrm{E}\left(W\left(\mathbf{x}_{k+1}\right) W\left(\mathbf{x}_{k}\right)\right) & =\mathrm{E}\left(W\left(\mathbf{x}_{k+1}\right)\right) \mathrm{E}\left(W\left(\mathbf{x}_{k}\right)\right)=0 .
\end{aligned}
$$

At any time instant $t_{k}$, the measurement taken by the agent can be written as

$$
y\left(\mathbf{x}_{k}\right)=F\left(\mathbf{x}_{k}\right)+w\left(\mathbf{x}_{k}\right) .
$$

The problem we are investigating into is whether the agent is able to explore the field $Y(\mathbf{x})$ based on its measurements $y\left(\mathbf{x}_{k}\right)$ and how difficult the field is to be explored. Examples of explorative behaviors can be climbing the local gradient, following a level curve, or simply increasing or decreasing the sensor measurements. To address this problem, we first introduce a concept of "false-walk".

Suppose $\mathbf{x}_{0} \in \mathbb{R}^{n}$ is a regular point of $F(\mathbf{x})$, which indicates that $d F\left(\mathbf{x}_{0}\right) \neq 0$, e.g. $\nabla F\left(\mathbf{x}_{0}\right) \neq 0$. Define the sets

$$
\begin{gathered}
U^{+}(\varepsilon)=\left\{\mathbf{x} \mid F(\mathbf{x})-F\left(\mathbf{x}_{0}\right)>\varepsilon\right\}, \\
U^{-}(\varepsilon)=\left\{\mathbf{x} \mid F(\mathbf{x})-F\left(\mathbf{x}_{0}\right)<-\varepsilon\right\}, \\
U^{0}(\varepsilon)=\left\{\mathbf{x}|| F(\mathbf{x})-F\left(\mathbf{x}_{0}\right) \mid \leq \varepsilon\right\} .
\end{gathered}
$$

And

$$
B\left(\delta_{1}\right)=\left\{\mathbf{x} \mid\left\|\mathbf{x}-\mathbf{x}_{0}\right\| \leq \delta_{1}\right\}
$$

where $\varepsilon>0$ and $\delta_{1}>0$ are two constants. We have the following definition.

Definition 2.1: A false-walk is a movement performed by a sensing agent from $\mathbf{x}_{0}$ to $\mathbf{x}$, such that one of the following conditions is satisfied:

1. If $\mathbf{x} \in U^{+}(\varepsilon)$, then $y(\mathbf{x}) \leq y\left(\mathbf{x}_{0}\right)+\varepsilon$;

2. If $\mathbf{x} \in U^{-}(\varepsilon)$, then $y(\mathbf{x}) \geq y\left(\mathbf{x}_{0}\right)-\varepsilon$;

3. If $\mathbf{x} \in U^{0}(\varepsilon)$, then $\left|y(\mathbf{x})-y\left(\mathbf{x}_{0}\right)\right|>\varepsilon$.

A false-walk happens when the changes of the sensor readings are not consistent with the changes of the mean field values. In a successful exploration mission, it is desired that the probability of false-walks (denoted by $\operatorname{Pr}(\mathrm{FW})$ ) to be small. To study the relationships between the probability of false-walks and the properties of a field, we propose the following definition of "local explorability".

Definition 2.2: Given $\varepsilon>0$ and $0<p<1$, we say the field $Y(\mathbf{x})$ is locally $(p, \varepsilon)$ explorable at $\mathbf{x}_{0}$ (or $\mathbf{x}_{0}$ is $(p, \varepsilon)$ explorable) if for $\forall \delta_{1}>0$ satisfying

$$
\left(U^{+}(\varepsilon) \cup U^{-}(\varepsilon)\right) \cap B\left(\delta_{1}\right) \neq \varnothing,
$$

the following conditions are satisfied:

1. If $\mathbf{x} \in U^{+}(\varepsilon) \cap B\left(\delta_{1}\right)$, then

$$
\operatorname{Pr}\left(Y(\mathbf{x})>Y\left(\mathbf{x}_{0}\right)+\varepsilon\right)>\frac{1+p}{2} ;
$$

2. If $\mathbf{x} \in U^{-}(\varepsilon) \cap B\left(\delta_{1}\right)$, then

$$
\operatorname{Pr}\left(Y(\mathbf{x})<Y\left(\mathbf{x}_{0}\right)-\varepsilon\right)>\frac{1+p}{2}
$$

3. If $\mathbf{x} \in U^{0}(\varepsilon) \cap B\left(\delta_{1}\right)$, then

$$
\operatorname{Pr}\left(\left|Y(\mathbf{x})-Y\left(\mathbf{x}_{0}\right)\right| \leq \varepsilon\right)>\frac{1+p}{2} .
$$

Moreover, $Y(\mathbf{x})$ is $(p, \varepsilon)$ explorable on an open set $C(\mathbf{x})$ where measurements are taken, s.t. for every $\mathbf{x}_{0} \in C(\mathbf{x}), Y(\mathbf{x})$ is locally $(p, \varepsilon)$ explorable at $\mathbf{x}_{0}$. We call $p$ the "explorable probability" and $\varepsilon$ the "resolution" of the sensor. The parameter $\delta_{1}$ represents the distance between the starting and ending positions of the agent.

Given the definitions of false-walk and local explorability, we now show that a field being $(p, \varepsilon)$ explorable is the sufficient and necessary condition for the probability of a false-walk $\operatorname{Pr}(F W)<\frac{1-p}{2}$.

Proposition 2.3: Given $\varepsilon>0$. Suppose an agent moves a distance $\delta_{1}$ from $\mathbf{x}_{0}$ to $\mathbf{x}$ in a randomly selected direction in a noisy field $Y(\mathbf{x})$. The distance $\delta_{1}$ satisfies $\left(U^{+}(\varepsilon) \cup U^{-}(\varepsilon)\right) \cap$ $B\left(\delta_{1}\right) \neq \varnothing$. The probability of a false-walk $\operatorname{Pr}(F W)<\frac{1-p}{2}$ if and only if the field is locally $(p, \varepsilon)$ explorable at $\mathbf{x}_{0}$.

Proof: We first prove the sufficient condition. Suppose $\mathbf{x}_{0}$ is $(p, \varepsilon)$ explorable. From the definition, if $\mathbf{x} \in U^{+}(\varepsilon) \cap$ $B\left(\delta_{1}\right), \operatorname{Pr}\left(Y(\mathbf{x})>Y\left(\mathbf{x}_{0}\right)+\varepsilon\right)>\frac{1+p}{2}$. Therefore, $\operatorname{Pr}(Y(\mathbf{x}) \leq$ $\left.Y\left(\mathbf{x}_{0}\right)+\varepsilon\right)<1-\frac{1+p}{2}=\frac{1-p}{2}$. Similarly, if $\mathbf{x} \in U^{-}(\varepsilon) \cap B\left(\delta_{1}\right)$, we can calculate that $\operatorname{Pr}\left(Y(\mathbf{x}) \geq Y\left(\mathbf{x}_{0}\right)-\varepsilon\right)<\frac{1-p}{2}$. And if $\mathbf{x} \in$ $U^{0}(\varepsilon) \cap B\left(\delta_{1}\right)$, we can obtain that $\operatorname{Pr}\left(\left|Y(\mathbf{x})-Y\left(\mathbf{x}_{0}\right)\right|>\varepsilon\right)<$ $\frac{1-p}{2}$. Therefore, the probability of a false-walk $\operatorname{Pr}(F W)<$ $\frac{1-p}{2}$ if $\mathbf{x}_{0}$ is $(p, \varepsilon)$ explorable.

For the necessary condition, suppose the probability of a false-walk $\operatorname{Pr}(F W)<\frac{1-p}{2}$. If $\mathbf{x} \in U^{+}(\varepsilon) \cap B\left(\delta_{1}\right)$, then the probability of a false-walk is $\operatorname{Pr}\left(Y(\mathbf{x}) \leq Y\left(\mathbf{x}_{0}\right)+\varepsilon\right)<$ $\frac{1-p}{2}$, which gives $\left.\operatorname{Pr}\left(Y(\mathbf{x})>Y\left(\mathbf{x}_{0}\right)+\varepsilon\right)\right)>\frac{1+p}{2}$. Similarly, if $\mathbf{x} \in U^{-}(\varepsilon) \cap B\left(\delta_{1}\right)$, then the probability of a false-walk is $\operatorname{Pr}\left(Y(\mathbf{x}) \geq Y\left(\mathbf{x}_{0}\right)-\varepsilon\right)<\frac{1-p}{2}$, which gives $\operatorname{Pr}\left(Y(\mathbf{x})<Y\left(\mathbf{x}_{0}\right)-\right.$ $\varepsilon))>\frac{1+p}{2}$. And if $\mathbf{x} \in U^{0}(\varepsilon) \cap B\left(\delta_{1}\right)$, then the probability of a false-walk is $\operatorname{Pr}\left(\left|Y(\mathbf{x})-Y\left(\mathbf{x}_{0}\right)\right|>\varepsilon\right)<\frac{1-p}{2}$, which gives $\operatorname{Pr}\left(\left|Y(\mathbf{x})-Y\left(\mathbf{x}_{0}\right)\right| \leq \varepsilon\right)>\frac{1+p}{2}$. According to the definition, the field is locally $(p, \varepsilon)$ explorable at $\mathbf{x}_{0}$.

\section{B. Explorable Fields}

Given the definition of local explorability, we want to study the relationships between the explorable probability $p$ and the noise properties of the field and in the measuring process.

Given two positions in the field $\mathbf{x}_{0}$ and $\mathbf{x}$, we can define a new random variable $Z(\mathbf{x})=W(\mathbf{x})-W\left(\mathbf{x}_{0}\right)$. Then we can calculate that

$$
\begin{aligned}
& \operatorname{Pr}\left(Y(\mathbf{x})<Y\left(\mathbf{x}_{0}\right)-\varepsilon\right) \\
& =\operatorname{Pr}\left(F(\mathbf{x})-F\left(\mathbf{x}_{0}\right)+W(\mathbf{x})-W\left(\mathbf{x}_{0}\right)<-\varepsilon\right) \\
& =\operatorname{Pr}\left(W(\mathbf{x})-W\left(\mathbf{x}_{0}\right)<-\varepsilon-\left(F(\mathbf{x})-F\left(\mathbf{x}_{0}\right)\right)\right) \\
& =\int_{-\infty}^{-\varepsilon-\left(F(\mathbf{x})-F\left(\mathbf{x}_{0}\right)\right)} f(z) d z
\end{aligned}
$$

where $f(z)$ is the p.d.f of the random variable $Z(\mathbf{x})$. Similarly,

$$
\operatorname{Pr}\left(Y(\mathbf{x})>Y\left(\mathbf{x}_{0}\right)+\varepsilon\right)=1-\int_{-\infty}^{\varepsilon-\left(F(\mathbf{x})-F\left(\mathbf{x}_{0}\right)\right)} f(z) d z .
$$


According to the definition, if $\mathbf{x} \in U^{-}(\varepsilon) \cap B\left(\delta_{1}\right)$, we require that $\operatorname{Pr}\left(Y(\mathbf{x})<Y\left(\mathbf{x}_{0}\right)-\varepsilon\right)>\frac{1+p}{2}$. And if $\mathbf{x} \in U^{+}(\varepsilon) \cap$ $B\left(\delta_{1}\right)$, we require that $\operatorname{Pr}\left(Y(\mathbf{x})>Y\left(\mathbf{x}_{0}\right)+\varepsilon\right)>\frac{1+p}{2}$. We can calculate the probability only when we know the p.d.f of the noises. Let's consider a specially case where the noise is i.i.d Gaussian.

Example 2.4: Suppose $W(\mathbf{x})$ is i.i.d Gaussian noise with zero mean. So $W(\mathbf{x}) \sim \mathscr{N}\left(0, \sigma^{2}\right)$. Hence, $Z(\mathbf{x}) \sim \mathscr{N}\left(0,2 \sigma^{2}\right)$.

When $\mathbf{x} \in U^{-}(\varepsilon) \cap B\left(\delta_{1}\right)$, we can calculate that

$$
\begin{aligned}
& \operatorname{Pr}\left(Y(\mathbf{x})<Y\left(\mathbf{x}_{0}\right)-\varepsilon\right) \\
& =\frac{1}{\sqrt{4 \pi \sigma^{2}}} \int_{-\infty}^{-\varepsilon-\left(F(\mathbf{x})-F\left(\mathbf{x}_{0}\right)\right)} e^{-\frac{z^{2}}{4 \sigma^{2}}} d z \\
& =\frac{1}{2}\left(1+\operatorname{erf}\left(\frac{-\varepsilon-\left(F(\mathbf{x})-F\left(\mathbf{x}_{0}\right)\right)}{2 \sigma}\right)\right)
\end{aligned}
$$

Similarly, when $\mathbf{x} \in U^{+}(\varepsilon) \cap B\left(\delta_{1}\right)$, we can derive

$$
\begin{aligned}
& \operatorname{Pr}\left(Y(\mathbf{x})>Y\left(\mathbf{x}_{0}\right)+\varepsilon\right) \\
& =1-\frac{1}{2}\left(1+\operatorname{erf}\left(\frac{\varepsilon-\left(F(\mathbf{x})-F\left(\mathbf{x}_{0}\right)\right)}{2 \sigma}\right)\right) .
\end{aligned}
$$

From the symmetry of the Gaussian distribution and the fact that $F(\mathbf{x})-F\left(\mathbf{x}_{0}\right)<-\varepsilon$ when $\mathbf{x} \in U^{-}(\varepsilon) \cap B\left(\delta_{1}\right)$ and $F(\mathbf{x})-F\left(\mathbf{x}_{0}\right)>\varepsilon$ when $\mathbf{x} \in U^{+}(\varepsilon) \cap B\left(\delta_{1}\right)$, we can see that the probabilities in equation (11) and (12) are larger than $\frac{1}{2}$, and as the variance $\sigma^{2}$ increases, the probabilities decrease. For the probability to be larger than $\frac{1+p}{2}, \sigma$ should satisfy

$$
\sigma<\frac{|\varepsilon-| F(\mathbf{x})-F\left(\mathbf{x}_{0}\right)||}{2 \operatorname{erf}^{-1}(p)}
$$

The above example verifies the intuition that when the noise strength gets lower, a higher explorable probability can be achieved. If we have the knowledge of the noise distribution and the sensor resolution $\varepsilon$, then from equation (13), we can check the explorable probability of a field at each position based on the field values $F(\mathbf{x})$ and $F\left(\mathbf{x}_{0}\right)$. In this way, we can tell if a field is difficult to be explored or not.

\section{REDUCING SENSOR READINGS}

In this section, we introduce and analyze an exploration algorithm that attempts to direct an agent to move in directions that reduce the sensor readings in the context of local explorability.

Let $C(\mathbf{x})$ where $\mathbf{x} \in \mathbb{R}^{2}$ be a bounded open set that contains only regular points of $F(\mathbf{x})$. Since $F(\mathbf{x})$ is assumed to be smooth, for the position $\mathbf{x}$ in the field, we have

$$
\lim _{\delta_{1} \rightarrow 0}\left(F\left(\mathbf{x}+\delta_{1} \mathbf{h}\right)-F(\mathbf{x})\right)=\lim _{\delta_{1} \rightarrow 0}\left(F(\mathbf{x})-F\left(\mathbf{x}-\delta_{1} \mathbf{h}\right)\right) .
$$

By the definition of the limit, the above equation is equivalent to given $\varepsilon_{2}>0$, there exists $\delta_{2}\left(\varepsilon_{2}\right)>0$, such that $\forall \delta_{1}<$ $\delta_{2}\left(\varepsilon_{2}\right)$, we have

$$
\left|\left(F\left(\mathbf{x}+\delta_{1} \mathbf{h}\right)-F(\mathbf{x})\right)-\left(F(\mathbf{x})-F\left(\mathbf{x}-\delta_{1} \mathbf{h}\right)\right)\right|<\varepsilon_{2} .
$$

The exploration algorithm is as follows:

Algorithm 3.1: Given $(p, \varepsilon)$, where $\varepsilon>0$ and $0<p<1$. Suppose a sensing agent is taking measurements of a noisy scalar field $Y(\mathbf{x})$ discretely. Set $\varepsilon_{2}=\frac{1}{8} \varepsilon p$, and choose $0<$ $\delta_{1}<\delta_{2}\left(\varepsilon_{2}\right)$.

$\mathrm{S} 1$. Let $i=0$. Set the initial position of the agent to be $\mathbf{x}_{0} \in C(\mathbf{x})$, and the agent takes a measurement $y\left(\mathbf{x}_{0}\right)$.

S2. The agent moves to a randomly selected direction from $\mathbf{x}_{0}$ to $\mathbf{x}_{1}$ for a distance of $\delta_{1}$ and obtains $y\left(\mathbf{x}_{1}\right)$. Set $i=1$.

S3. Define the unit direction vector $\mathbf{h}=\frac{\mathbf{x}_{i}-\mathbf{x}_{i-1}}{\left\|\mathbf{x}_{i}-\mathbf{x}_{i-1}\right\|}$.

1. If $y\left(\mathbf{x}_{i}\right)<y\left(\mathbf{x}_{i-1}\right)-\varepsilon$, the agent moves along the direction of $\mathbf{h}$ for a distance of $\delta_{1}$ and reaches $\mathbf{x}_{i+1}$.

2. If $y\left(\mathbf{x}_{i}\right)>y\left(\mathbf{x}_{i-1}\right)+\varepsilon$, the agent moves along the direction of $-\mathbf{h}$ for a distance of $\delta_{1}$ and reaches $\mathbf{x}_{i+1}$.

3. If $\left|y\left(\mathbf{x}_{i}\right)-y\left(\mathbf{x}_{i-1}\right)\right| \leq \varepsilon$, the agent randomly selects a direction $\mathbf{h}_{\perp}$, which satisfies $\mathrm{E}\left(\mathbf{h} \cdot \mathbf{h}_{\perp}\right)=0$ and moves a distance of $\delta_{1}$ along it to reach $\mathbf{x}_{i+1}$. The probability distribution of this random selection satisfies $\operatorname{Pr}\left(\mathbf{x} \in \mathrm{U}^{+}(\varepsilon) \cup \mathbf{x} \in \mathrm{U}^{-}(\varepsilon)\right)>\frac{1}{2}$.

S4. Let $i=i+1$, go to step S3.

Based on the above algorithm, we have the following proposition.

Proposition 3.2: Consider a noisy field $Y(\mathbf{x})$ where $\mathbf{x} \in$ $\mathbb{R}^{2}$. Suppose a sensing agent is moving in the field according to Algorithm (3.1). If

1. The positions on the trajectory $\left\{\mathbf{x}_{i}\right\}, i \in(1,2, \cdots)$ are $(p, \varepsilon)$ explorable.

2. When $\mathbf{x}_{i} \in U^{0}(\varepsilon) \cap B\left(\delta_{1}\right)$, we have

$$
\left|\operatorname{Pr}\left(Y\left(\mathbf{x}_{i}\right)<Y\left(\mathbf{x}_{i-1}\right)-\varepsilon\right)-\operatorname{Pr}\left(Y\left(\mathbf{x}_{i}\right)>Y\left(\mathbf{x}_{i-1}\right)+\varepsilon\right)\right|<\frac{1}{2} p .
$$

then

$$
\operatorname{Pr}\left(\mathrm{E}\left(Y\left(\mathbf{x}_{i+1}\right) \mid y\left(\mathbf{x}_{i}\right)\right)<y\left(\mathbf{x}_{i}\right)\right)>\frac{1}{2} .
$$

Note that in the proposition, $\mathrm{E}(\cdot)$ represents the expectation of a random variable.

Proof: Since the measurement of the agent at step $i+1$ depends on the measurements at steps $i$ and $i-1$, based on the law of total expectation, the conditional expectation $\mathrm{E}\left(Y\left(\mathbf{x}_{i+1}\right) \mid y\left(\mathbf{x}_{i}\right)\right)$ can be written as

$$
\begin{aligned}
& \mathrm{E}\left(Y\left(\mathbf{x}_{i+1}\right) \mid y\left(\mathbf{x}_{i}\right)\right) \\
& =\mathrm{E}\left(Y\left(\mathbf{x}_{i+1}\right) \mid y\left(\mathbf{x}_{i}\right), y\left(\mathbf{x}_{i-1}\right)>y\left(\mathbf{x}_{i}\right)+\varepsilon\right) \operatorname{Pr}\left(Y\left(\mathbf{x}_{i-1}\right)>Y\left(\mathbf{x}_{i}\right)+\varepsilon\right) \\
& +\mathrm{E}\left(Y\left(\mathbf{x}_{i+1}\right) \mid y\left(\mathbf{x}_{i}\right), y\left(\mathbf{x}_{i-1}\right)<y\left(\mathbf{x}_{i}\right)-\varepsilon\right) \operatorname{Pr}\left(Y\left(\mathbf{x}_{i-1}\right)<Y\left(\mathbf{x}_{i}\right)-\varepsilon\right) \\
& +\mathrm{E}\left(Y\left(\mathbf{x}_{i+1}\right) \mid y\left(\mathbf{x}_{i}\right), y\left(\mathbf{x}_{i}\right)-\varepsilon \leq y\left(\mathbf{x}_{i-1}\right) \leq y\left(\mathbf{x}_{i}\right)+\varepsilon\right) \\
& \cdot \operatorname{Pr}\left(Y\left(\mathbf{x}_{i}\right)-\varepsilon \leq Y\left(\mathbf{x}_{i-1}\right) \leq Y\left(\mathbf{x}_{i}\right)+\varepsilon\right) .
\end{aligned}
$$

For simplicity, we denote the event $Y\left(\mathbf{x}_{i-1}\right)>Y\left(\mathbf{x}_{i}\right)+$ $\varepsilon$ as $\mathrm{e}_{1}, \quad Y\left(\mathbf{x}_{i-1}\right)<Y\left(\mathbf{x}_{i}\right)-\varepsilon$ as $\mathrm{e}_{2}$ and $Y\left(\mathbf{x}_{i}\right)-$ $\varepsilon \leq Y\left(\mathbf{x}_{i-1}\right) \leq Y\left(\mathbf{x}_{i}\right)+\varepsilon$ as $\mathrm{e}_{3}$. Then the above equation can be re-written as

$$
\begin{aligned}
& \mathrm{E}\left(Y\left(\mathbf{x}_{i+1}\right) \mid y\left(\mathbf{x}_{i}\right)\right)=\mathrm{E}\left(Y\left(\mathbf{x}_{i+1}\right) \mid y\left(\mathbf{x}_{i}\right), \mathrm{e}_{1}\right) \operatorname{Pr}\left(\mathrm{e}_{1}\right) \\
& +\mathrm{E}\left(Y\left(\mathbf{x}_{i+1}\right) \mid y\left(\mathbf{x}_{i}\right), \mathrm{e}_{2}\right) \operatorname{Pr}\left(\mathrm{e}_{2}\right)+\mathrm{E}\left(Y\left(\mathbf{x}_{i+1}\right) \mid y\left(\mathbf{x}_{i}\right), \mathrm{e}_{3}\right) \operatorname{Pr}\left(\mathrm{e}_{3}\right) .
\end{aligned}
$$

Since we have

$$
\begin{aligned}
Y\left(\mathbf{x}_{i+1}\right) & =F\left(\mathbf{x}_{i+1}\right)+W\left(\mathbf{x}_{i+1}\right), \\
y\left(\mathbf{x}_{i}\right) & =F\left(\mathbf{x}_{i}\right)+w\left(\mathbf{x}_{i}\right) .
\end{aligned}
$$


Therefore,

$$
Y\left(\mathbf{x}_{i+1}\right)=y\left(\mathbf{x}_{i}\right)+F\left(\mathbf{x}_{i+1}\right)-F\left(\mathbf{x}_{i}\right)+W\left(\mathbf{x}_{i+1}\right)-w\left(\mathbf{x}_{i}\right),
$$

We discuss three cases separately.

1. If $y\left(\mathbf{x}_{i-1}\right)>y\left(\mathbf{x}_{i}\right)+\varepsilon$, according to the algorithm, the agent moves along the direction of $\mathbf{h}$ for $\delta_{1}$ and reaches $\mathbf{x}_{i+1}$. Hence,

$$
F\left(\mathbf{x}_{i+1}\right)=F\left(\mathbf{x}_{i}+\delta_{1} \mathbf{h}\right) .
$$

Then

$$
\mathrm{E}\left(Y\left(\mathbf{x}_{i+1}\right) \mid y\left(\mathbf{x}_{i}\right), \mathrm{e}_{1}\right)=y\left(\mathbf{x}_{i}\right)+F\left(\mathbf{x}_{i}+\delta_{1} \mathbf{h}\right)-F\left(\mathbf{x}_{i}\right)-w\left(\mathbf{x}_{i}\right) .
$$

2. If $y\left(\mathbf{x}_{i-1}\right)<y\left(\mathbf{x}_{i}\right)-\varepsilon$, the agent moves along the direction of $-\mathbf{h}$ for $\delta_{1}$ and reaches $\mathbf{x}_{i+1}$. So

$$
F\left(\mathbf{x}_{i+1}\right)=F\left(\mathbf{x}_{i}-\delta_{1} \mathbf{h}\right) .
$$

Similarly, we can obtain

$$
\mathrm{E}\left(Y\left(\mathbf{x}_{i+1}\right) \mid y\left(\mathbf{x}_{i}\right), \mathrm{e}_{2}\right)=y\left(\mathbf{x}_{i}\right)+F\left(\mathbf{x}_{i}-\delta_{1} \mathbf{h}\right)-F\left(\mathbf{x}_{i}\right)-w\left(\mathbf{x}_{i}\right) .
$$

3. If $y\left(\mathbf{x}_{i}\right)-\varepsilon \leq y\left(\mathbf{x}_{i-1}\right) \leq y\left(\mathbf{x}_{i}\right)+\varepsilon$, the agent randomly selects $\mathbf{h}_{\perp}$ satisfying $\mathrm{E}\left(\mathbf{h} \cdot \mathbf{h}_{\perp}\right)=0$ and moves along it to reach $\mathbf{x}_{i+1}$. Hence,

$\mathrm{E}\left(Y\left(\mathbf{x}_{i+1}\right) \mid y\left(\mathbf{x}_{i}\right), \mathrm{e}_{3}\right)=y\left(\mathbf{x}_{i}\right)+\mathrm{E}\left(F\left(\mathbf{x}_{i}+\delta_{1} \mathbf{h}_{\perp}\right)\right)-F\left(\mathbf{x}_{i}\right)-w\left(\mathbf{x}_{i}\right)$.

In $2 \mathrm{D}$, there are only two choices of $\mathbf{h}_{\perp}$, which can be denoted by $\mathbf{h}_{\perp}^{+}$and $\mathbf{h}_{\perp}^{-}$, where $\mathbf{h}_{\perp}^{+}$is to the right of $\mathbf{h}_{\perp}$ and $\mathbf{h}_{\perp}^{-}$is to the left of $\mathbf{h}_{\perp}$. The probability of the choices are $\operatorname{Pr}\left(\mathbf{h}_{\perp}^{+}\right)=\operatorname{Pr}\left(\mathbf{h}_{\perp}^{-}\right)=\frac{1}{2}$. Note that $\mathbf{h}_{\perp}^{+}=-\mathbf{h}_{\perp}^{-}$. Therefore, we can calculate that

$$
\begin{aligned}
& \mathrm{E}\left(F\left(\mathbf{x}_{i}+\delta_{1} \mathbf{h}_{\perp}\right)\right) \\
& =F\left(\mathbf{x}_{i}+\delta_{1} \mathbf{h}_{\perp}^{+}\right) \operatorname{Pr}\left(\mathbf{h}_{\perp}^{+}\right)+F\left(\mathbf{x}_{i}+\delta_{1} \mathbf{h}_{\perp}^{-}\right) \operatorname{Pr}\left(\mathbf{h}_{\perp}^{-}\right) \\
& =\frac{1}{2} F\left(\mathbf{x}_{i}+\delta_{1} \mathbf{h}_{\perp}^{+}\right)+\frac{1}{2} F\left(\mathbf{x}_{i}-\delta_{1} \mathbf{h}_{\perp}^{+}\right)=F\left(\mathbf{x}_{i}\right),
\end{aligned}
$$

which gives

$$
\mathrm{E}\left(Y\left(\mathbf{x}_{i+1}\right) \mid y\left(\mathbf{x}_{i}\right), \mathrm{e}_{3}\right)=y\left(\mathbf{x}_{i}\right)-w\left(\mathbf{x}_{i}\right) .
$$

Combine the three cases together, we have

$$
\begin{aligned}
& \mathrm{E}\left(Y\left(\mathbf{x}_{i+1}\right) \mid y\left(\mathbf{x}_{i}\right)\right) \\
= & \left(y\left(\mathbf{x}_{i}\right)+F\left(\mathbf{x}_{i}+\delta_{1} \mathbf{h}\right)-F\left(\mathbf{x}_{i}\right)-w\left(\mathbf{x}_{i}\right)\right) \operatorname{Pr}\left(\mathrm{e}_{1}\right) \\
& +\left(y\left(\mathbf{x}_{i}\right)+F\left(\mathbf{x}_{i}-\delta_{1} \mathbf{h}\right)-F\left(\mathbf{x}_{i}\right)-w\left(\mathbf{x}_{i}\right)\right) \operatorname{Pr}\left(\mathrm{e}_{2}\right) \\
& +\left(y\left(\mathbf{x}_{i}\right)-w\left(\mathbf{x}_{i}\right)\right) \operatorname{Pr}\left(\mathrm{e}_{3}\right) \\
= & y\left(\mathbf{x}_{i}\right)-w\left(\mathbf{x}_{i}\right)+\left(F\left(\mathbf{x}_{i}+\delta_{1} \mathbf{h}\right)-F\left(\mathbf{x}_{i}\right)\right) \operatorname{Pr}\left(\mathrm{e}_{1}\right) \\
& -\left(F\left(\mathbf{x}_{i}\right)-F\left(\mathbf{x}_{i}-\delta_{1} \mathbf{h}\right)\right) \operatorname{Pr}\left(\mathrm{e}_{2}\right) .
\end{aligned}
$$

Based on equation (15), we can calculate that for $\delta_{1}<$ $\delta_{2}\left(\varepsilon_{2}\right)$, equation (29) becomes

$$
\begin{aligned}
& \mathrm{E}\left(Y\left(\mathbf{x}_{i+1}\right) \mid y\left(\mathbf{x}_{i}\right)\right) \\
& <y\left(\mathbf{x}_{i}\right)-w\left(\mathbf{x}_{i}\right)+\left(F\left(\mathbf{x}_{i}\right)-F\left(\mathbf{x}_{i}-\delta_{1} \mathbf{h}\right)\right)\left(\operatorname{Pr}\left(\mathrm{e}_{1}\right)-\operatorname{Pr}\left(\mathrm{e}_{2}\right)\right) \\
& +K \varepsilon_{2} \operatorname{Pr}\left(\mathrm{e}_{1}\right) \\
& =y\left(\mathbf{x}_{i}\right)-w\left(\mathbf{x}_{i}\right)+A+B
\end{aligned}
$$

where $A=\left(F\left(\mathbf{x}_{i}\right)-F\left(\mathbf{x}_{i}-\delta_{1} \mathbf{h}\right)\right)\left(\operatorname{Pr}\left(\mathrm{e}_{1}\right)-\operatorname{Pr}\left(\mathrm{e}_{2}\right)\right), \quad B=$ $K \varepsilon_{2} \operatorname{Pr}\left(\mathrm{e}_{1}\right)$ and $-1<K<1$. Therefore,

$$
\begin{aligned}
\operatorname{Pr}\left(\mathrm{E}\left(Y\left(\mathbf{x}_{i+1}\right) \mid y\left(\mathbf{x}_{i}\right)\right)<y\left(\mathbf{x}_{i}\right)\right) & =\operatorname{Pr}\left(A+B-W\left(\mathbf{x}_{i}\right)<0\right) \\
& =\operatorname{Pr}\left(W\left(\mathbf{x}_{i}\right)>A+B\right) .
\end{aligned}
$$

There are also three cases.

1. If $\mathbf{x}_{i-1} \in U^{+}(\varepsilon)$, which means $F\left(\mathbf{x}_{i}\right)-F\left(\mathbf{x}_{i-1}\right)<-\varepsilon$, then according to the definition, $\operatorname{Pr}\left(\mathrm{e}_{1}\right)>\frac{1+p}{2}$ and $\operatorname{Pr}\left(\mathrm{e}_{2}\right)<$ $1-\frac{1+p}{2}$. Therefore, $\operatorname{Pr}\left(\mathrm{e}_{1}\right)-\operatorname{Pr}\left(\mathrm{e}_{2}\right)>p$. Hence

$$
A+B<-\varepsilon p+K \varepsilon_{2} \operatorname{Pr}\left(\mathrm{e}_{1}\right) .
$$

2. If $\mathbf{x}_{i-1} \in U^{-}(\varepsilon)$, which means $F\left(\mathbf{x}_{i}\right)-F\left(\mathbf{x}_{i-1}\right)>\varepsilon$, then $\operatorname{Pr}\left(\mathrm{e}_{2}\right)>\frac{1+p}{2}$ and $\operatorname{Pr}\left(\mathrm{e}_{1}\right)<1-\frac{1+p}{2}$. Therefore, $\operatorname{Pr}\left(\mathrm{e}_{1}\right)-$ $\operatorname{Pr}\left(\mathrm{e}_{2}\right)<-p$. We can also obtain $A+B<-\varepsilon p+K \varepsilon_{2} \operatorname{Pr}\left(\mathrm{e}_{1}\right)$.

3. If $\mathbf{x}_{i-1} \in U^{0}(\varepsilon)$, which means $\left|F\left(\mathbf{x}_{i}\right)-F\left(\mathbf{x}_{i-1}\right)\right| \leq \varepsilon$. Then $\left.\mid \operatorname{Pr}\left(\mathrm{e}_{1}\right)\right)-\operatorname{Pr}\left(\mathrm{e}_{2}\right) \mid<\frac{1}{2} p$. Hence

$$
-\frac{1}{2} p \varepsilon+K \varepsilon_{2} \operatorname{Pr}\left(\mathrm{e}_{1}\right)<A+B<\frac{1}{2} p \varepsilon+K \varepsilon_{2} \operatorname{Pr}\left(\mathrm{e}_{1}\right) .
$$

Considering the three cases and based on the law of total probability, we can obtain

$$
\begin{aligned}
A+B & =\mathrm{E}\left(A+B \mid \mathbf{x}_{i} \in U^{-}(\varepsilon)\right) \operatorname{Pr}\left(\mathbf{x}_{i} \in U^{-}(\varepsilon)\right) \\
& +\mathrm{E}\left(A+B \mid \mathbf{x}_{i} \in U^{+}(\varepsilon)\right) \operatorname{Pr}\left(\mathbf{x}_{i} \in U^{+}(\varepsilon)\right) \\
& +\mathrm{E}\left(A+B \mid \mathbf{x}_{i} \in U^{0}(\varepsilon)\right) \operatorname{Pr}\left(\mathbf{x}_{i} \in U^{0}(\varepsilon)\right) \\
& <-\varepsilon p\left(\operatorname{Pr}\left(\mathbf{x}_{i} \in U^{-}(\varepsilon)\right)+\operatorname{Pr}\left(\mathbf{x}_{i} \in U^{+}(\varepsilon)\right)\right) \\
& +\frac{1}{2} p \varepsilon \operatorname{Pr}\left(\mathbf{x}_{i} \in U^{0}(\varepsilon)\right)+K \varepsilon_{2} \operatorname{Pr}\left(\mathrm{e}_{1}\right) .
\end{aligned}
$$

From the algorithm, we have $\operatorname{Pr}\left(\mathbf{x}_{i} \in U^{-}(\varepsilon)\right)+\operatorname{Pr}\left(\mathbf{x}_{i} \in\right.$ $\left.U^{+}(\varepsilon)\right)>\frac{1}{2}$, which gives $\operatorname{Pr}\left(\mathbf{x}_{i} \in U^{0}(\varepsilon)\right)<\frac{1}{2}$. We also have $K \operatorname{Pr}\left(\mathrm{e}_{1}\right)<1$. Plug $\varepsilon_{2}=\frac{1}{8} \varepsilon p$ into equation (34), we can obtain

$$
A+B<-\frac{1}{2} \varepsilon p+\frac{1}{4} \varepsilon p+\frac{1}{8} \varepsilon p=-\frac{1}{8} \varepsilon p .
$$

Since the noise term $w\left(\mathbf{x}_{i}\right)$ is zero mean, therefore

$$
\operatorname{Pr}\left(W\left(\mathbf{x}_{i}\right)>A+B\right)>\operatorname{Pr}\left(W\left(\mathbf{x}_{i}\right)>-\frac{1}{8} \varepsilon p\right)>\frac{1}{2} .
$$

Therefore,

$$
\operatorname{Pr}\left(\mathrm{E}\left(Y\left(\mathbf{x}_{k+1}\right) \mid y_{k}\right)<y\left(\mathbf{x}_{k}\right)\right)>\frac{1}{2} .
$$

According to Proposition 3.2, at each time instant, the probability of an agent moving in a direction that would reduce the sensor reading is larger than $\frac{1}{2}$ if the current position of the agent is $(p, \varepsilon)$ explorable.

\section{COOPERATIVE FILTERING AND EXPLORABILITY}

The concept of local explorability can be applied to determine how many agents are necessary to cooperatively explore a noisy field. As discussed previously, if at each time instant, one measurement $y(\mathbf{x})$ is taken at the position $\mathbf{x}$, the explorable probability $p$ varies with the change of the noise variance $\sigma^{2}$. As $\sigma^{2}$ increases, the explorable probability decreases. Suppose we are using $N$ agents forming a formation to take measurements of the field and produce the estimates 
of the field value at the formation center with a cooperative filter [6]. Intuitively, the estimated field values should contain less noises compared to the direct measurements at the formation center, which means the noise variance $\sigma^{\prime 2}$ that is present to the formation is less than $\sigma^{2}$. Consequently, the explorable probability $p$ should increase.

To verify the intuition, let's take a look at an example described in [6]. Suppose a cooperative Kalman filter is computed by a group of $N$ agents forming a formation. The agents are taking measurements of the field discretely. Denote the position of the $i$ th agent at the $k$ th time step as $\mathbf{r}_{i, k}$, the measurement taken by the agent as $p_{i, k}$, where $i=1, \cdots, N$. Define the position of the formation center at the $k$ th step as $\mathbf{r}_{c, k}=\frac{1}{N} \sum \mathbf{r}_{i, k}$ and the field value at the position center as $z_{c, k}$. Choose the state to be $\mathbf{s}_{k}=$ $\left(z_{c, k}, \nabla z_{c, k}^{T}\right)^{T}$ where $\nabla z_{c, k}$ is the gradient at the formation center. Define $A_{k-1}=\left(\begin{array}{cc}1 & \left(\mathbf{r}_{c, k}-\mathbf{r}_{c, k-1}\right)^{T} \\ 0 & \mathbf{I}_{3 \times 3}\end{array}\right)$ and $\mathbf{h}_{k-1}=$ $\left(0, E\left[H_{c, k-1}\left(\mathbf{r}_{c, k}-\mathbf{r}_{c, k-1}\right)\right]^{T}\right)^{T}$, where $H_{c, k-1}$ is the estimate of the Hessian at the formation center. Let $C_{k}$ be the $N \times 3$ matrix with its $i$ th row defined by $\left[1,\left(\mathbf{r}_{i, k}-\mathbf{r}_{c, k}\right)^{T}\right]$ for $i=1,2, \ldots, N$ and $D_{k}$ be the $N \times 4$ matrix with its $i$ th row vector defined by the Kronecker product $\frac{1}{2}\left(\left(\mathbf{r}_{i, k}-\mathbf{r}_{c, k}\right) \otimes\right.$ $\left.\left(\mathbf{r}_{i, k}-\mathbf{r}_{c, k}\right)\right)^{T}$. Define the $N \times 1$ measurement vector $\mathbf{p}_{k}=$ $\left[p_{i, k}\right]$. Then we can write down the state equation and the measurement equation:

$$
\begin{aligned}
\mathbf{s}_{k} & =A_{k-1} \mathbf{s}_{k-1}+\mathbf{h}_{k-1}+\mathbf{v}_{k-1}, \\
\mathbf{p}_{k} & =C_{k} \mathbf{s}_{k}+D_{k} \vec{H}_{c, k}+\mathbf{w}_{k} .
\end{aligned}
$$

where $\mathbf{v}_{k-1}$ is the noise vector which accounts for positioning errors, estimation errors for the Hessians, and errors caused by higher-order terms omitted from the Taylor expansion. $\mathbf{w}_{k}$ represents the measurement noise vector. Based on the state equation and the measurement equation, a cooperative Kalman filter can be constructed. Readers can refer to [6] for details.

Let $V_{k}=E\left[\mathbf{v}_{k} \mathbf{v}_{k}^{T}\right]$, and $W_{k}=E\left[\mathbf{w}_{k} \mathbf{w}_{k}^{T}\right]$. We can calculate that, as $k \rightarrow \infty$, the error covariance matrix satisfies

$$
P^{-1}=\left[A P A^{T}+V\right]^{-1}+C^{T} W^{-1} C .
$$

For simplicity, we drop the subscript $\infty$ in the above equation and in the following expressions.

Suppose $v(\mathbf{x})$ and $w(\mathbf{x})$ are i.i.d Gaussian noises with zero mean and variances $\sigma_{1}^{2}$ and $\sigma_{2}^{2}$. As $k \rightarrow \infty, A_{k} \rightarrow I_{3 \times 3}, V_{k} \rightarrow$ $\sigma_{1}^{2} I_{3 \times 3}$ and $W_{k} \rightarrow \sigma_{2}^{2} I_{3 \times 3}$. Assume that the agents form a symmetric formation and the distance from each agent to the formation center is $a$, which means $\left\|\mathbf{r}_{i, k}-\mathbf{r}_{c, k}\right\|=a$. Then we can calculate that [23]

$$
C^{T} W^{-1} C=\frac{1}{\sigma_{2}^{2}} \operatorname{diag}\left(N, \frac{1}{2} a^{2} N, \frac{1}{2} a^{2} N\right) .
$$

Hence,

$$
\begin{aligned}
P & =\operatorname{diag}\left(-\frac{1}{2} \sigma_{1}^{2}+\frac{\sigma_{1}}{2} \sqrt{\sigma_{1}^{2}+\frac{4 \sigma_{2}^{2}}{N}},\right. \\
& \left.-\frac{1}{2} \sigma_{1}^{2}+\frac{\sigma_{1}}{2} \sqrt{\sigma_{1}^{2}+\frac{8 \sigma_{2}^{2}}{a^{2} N}},-\frac{1}{2} \sigma_{1}^{2}+\frac{\sigma_{1}}{2} \sqrt{\sigma_{1}^{2}+\frac{8 \sigma_{2}^{2}}{a^{2} N}}\right) \\
& =\operatorname{diag}(P(1), P(2), P(3)),
\end{aligned}
$$

where $P(1)$ can be considered as the error covariance of the field value estimation at the formation center and $P(2)$ and $P(3)$ correspond to the error covariance of the field gradient estimation at the formation center. Suppose we have $\sigma_{1}=\frac{1}{2} \sigma_{2}$. Then we need to compare $\sigma^{\prime 2}=P(1)$ in the cooperative exploration case with $\sigma^{2}=\sigma_{2}^{2}$ in the single agent case as described in Section II-B. According to equation (13), if $\sigma^{\prime}<\sigma$, a higher explorable probability $p$ can be achieved. We can tell from equation (42) that when the number of agents $N$ increases, the trace of the error covariance matrix $P$ decreases. Consequently, we can achieve a higher explorable probability with more agents.

Example 4.1: Suppose we are using $N$ agents forming a symmetric formation to take measurements in a field $F(x, y)=x^{2}+y^{2}$ with $v \sim \mathscr{N}\left(0, \sigma_{1}^{2}\right)$ and $w \sim \mathscr{N}\left(0, \sigma_{2}^{2}\right)$. We would like to determine the minimum value of $N$. Assume that we know $\sigma_{1}=0.8$ and $\sigma_{2}=1$.6. If we choose $\varepsilon=1$, and choose two points in the field $\mathbf{x}_{0}=[1,1]^{T}$ and $\mathbf{x}=[1.1,1.1]^{T}$ as the consecutive positions of the formation center, then $F(\mathbf{x})-F\left(\mathbf{x}_{0}\right)=2.42$. Suppose we want the explorable probability to be larger than 0.95 , which means $p>0.9$. Then according to equation (13), we can calculate that $\sigma^{\prime}$ should satisfy $\sigma^{\prime}<0.8663$. If we only use one agent, $\sigma=\sigma_{2}=1.6$, which means we can not achieve 0.95 explorable probability. However, according to equation (42), we can calculate that when $N>1.5701, P(1)<0.8663^{2}$. Therefore, we can deploy $N \geq 2$ agents in the field to perform the exploration task.

\section{Simulation Results}

To justify our algorithm, we compare one agent and a group of three collaborative agents moving in a noisy scalar field according to Algorithm 3.1. The field is generated by the equation $Y(x, y)=(x-10)^{2}+2(y-10)^{2}+W(x, y)$ where the noise term $W(x, y)$ is i.i.d Gaussian with zero mean and variance $\sigma^{2}=1.6^{2}$. In the cooperative exploration case, the variance of the modeling error $v(x, y)$ is assumed to have $\sigma_{1}=0.8$. If we choose $\varepsilon=1$ and $p=0.9$, then according to the algorithm, we can set $\varepsilon_{2}=\frac{1}{8} \varepsilon p$, and $\delta_{2}(\varepsilon)=K \varepsilon$ where $K=1$. Then we can choose the step size $\delta_{1}=\delta_{2}(\varepsilon)=\frac{1}{8} \varepsilon p$.

Fig. 1 and Fig. 2 show the trajectories of a single agent and the center of the formation formed by three agents, respectively. Fig. 3 indicates the measurements taken by the single agent (the red line) and the estimates of the field value at the formation center (the blue line). We can tell from Fig. 3 that, under the same settings, multiple agents that are performing cooperative exploration converge faster than a single agent. 


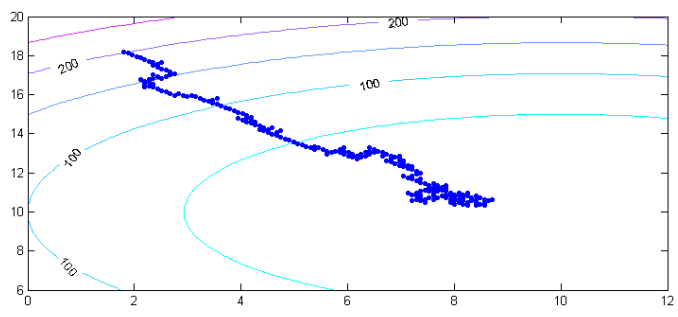

Fig. 1. The trajectory of a sensing agent.

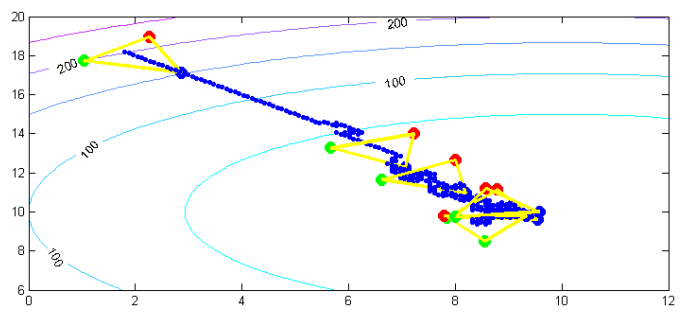

Fig. 2. The trajectory of the center of three agents.

To verify Algorithm 3.1, we can obtain the differences of the measurements between two adjacent steps, which are denoted by $y_{d}(i)=y(i)-y(i-1)$, where $i=1, \cdots$. We count the number of occasions that $y_{d}(i)<0$, and calculate the probability that $\operatorname{Pr}\left(y_{d}(i)<0\right)$. From Fig. 3, we can tell that the sensor readings of the single agent converge in around 400 steps. We obtain that there are 234 out of 400 steps that $y_{d}(i)<0$, which gives that $\operatorname{Pr}\left(y_{d}(i)<0\right)=0.585>0.5$. In the cooperative exploration case, the estimates of the field values at the formation center converge in around 200 steps. And in 138 out of 200 steps, $y_{d}(i)<0$, which gives that $\operatorname{Pr}\left(y_{d}(i)<0\right)=0.69>0.5$. This fact also justifies that the cooperative exploration using multiple agents increases the explorative probability.

\section{CONCLUSIONS}

We provide a definition of local explorability of a field which is used to analyze the tendency that a field will induce false-walks for a sensing agent. The relationships between the explorable probability and the noise properties of a field are studied. We also discuss the noise reduction capabilities of using multiple agents to explore a field, and the relationships between the number of agents and the explorable probability.

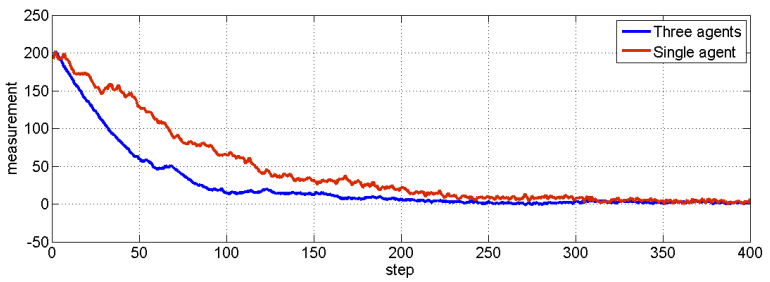

Fig. 3. Measurements of the field taken by a single agent (red line) and estimated field values at the center of a formation (blue line).

\section{REFERENCES}

[1] P. Ogren, E. Fiorelli, and N. E. Leonard, "Cooperative control of mobile sensor networks: Adaptive gradient climbing in a distributed environment," IEEE Transactions on Automatic Control, vol. 49, no. 8, pp. 1292-1302, 2004.

[2] J. Cortes, "Distributed gradient ascent of random fields by robotic sensor networks," in Proc. 46th IEEE Conf. on Decision and Control, 2007, pp. 3120-3126.

[3] E. Biyik and M. Arcak., "Gradient climbing in formation via extremum seeking and passivity-based coordination rules," in Proc. of 46th IEEE Conf. on Decision and Control, 2007, pp. 3133-3138.

[4] M. Krstic and H. H. Wang, "Stability of extremum seeking feedback for general dynamic systems," Automatica, vol. 36, pp. 595-601, 2000.

[5] M. Kemp, A. L. Bertozzi, and D. Marthaler, "Multi-UUV perimeter surveillance," in Proc. of the 2004 IEEE/OES Workshop on Autonomous Underwater Vehicles, 2004, pp. 102-107.

[6] F. Zhang and N. E. Leonard, "Cooperative control and filtering for cooperative exploration," IEEE Transactions on Automatic Control, vol. 55 , no. 3, pp. 650-663, 2010.

[7] W. Wu and F. Zhang, "Cooperative exploration of level surfaces of three dimentional scalar fields," Automatica, vol. 47(9), pp. 20442051, 2011.

[8] R. Ghabcheloo, A. P. Aguiar, A. Pascoal, C. Silvestre, I. Kaminer, and J. Hespanha, "Coordinated path-following in the presence of communication losses and time delays," SIAM, Journal on Control and Optimization, vol. 48, no. 1, pp. 234-265, 2009.

[9] S. Susca, S. Martínez, and F. Bullo, "Monitoring environmental boundaries with a robotic sensor network," IEEE Transactions on Control Systems Technology, vol. 16, no. 2, pp. 288-296, 2008.

[10] Z. Jin and A. L. Bertozzi, "Environmental boundary tracking and estimation using multiple autonomous vehicles," in Proc. of 46th IEEE Conf. on Decision and Control, 2007, pp. 4918-4923.

[11] E. Burian, D. Yoerger, A. Bradley, and H. Singh, "Gradient search with autonomous underwater vehicles using scalar measurements," in Proceedings of the 1996 Symposium on Autonomous Underwater Vehicle Technology, 1996, pp. 86 - 98.

[12] C. Zhang, D. Arnold, N. Ghods, A. Siranosian, and M. Krstic, "Source seeking with nonholonomic unicycle without position measurement and with tuning of forward velocity," Systems and Control Letters, vol. 56, pp. 245-252, 2007.

[13] C. G. Mayhew, R. G. Sanfelice, and A. R. Teel, "Robust hybrid source-seeking algorithms based on directional derivatives and their approximations," in Proc. of 47th IEEE Conf. Decision and Control, 2008, pp. 1735-1740.

[14] J. Cochran and M. Krstic, "Nonholonomic source seeking with tuning of angular velocity," IEEE Transactions on Automatic Control, vol. 54, no. 4, pp. 717-731, 2009.

[15] W. H. Moase, C. Manzie, and M. J. Brear, "Newton-like extremumseeking part I: Theory," in Proc. of 48th IEEE Conf. on Decision and Control, 2009, pp. 3839-3844.

[16] W. Burgard, M. Moors, C. Stachniss, and F. E. Schneider, "Coordinated multi-robot exploration," vol. 21, no. 3, pp. 376-386, 2005.

[17] B. Grocholsky, J. Keller, V. Kumar, and G. Pappas, "Cooperative air and ground surveillance," IEEE Robotics and Automation Magazine, vol. 13, no. 3, pp. 16-25, 2006.

[18] R. Simmons, D. Apfelbaum, W. Burgard, D. Fox, M. Moors, S. Thrun, and H. Younes, "Coordination for multi-robot exploration and mapping," in Proceedings Seventeenth National Conference on Artificial Intelligence (AAAI-2000), 2000, pp. 852-858.

[19] D. Fox, J. Ko, K. Konolige, B. Limketkai, D. Schulz, and B. Stewart, "Distributed multirobot exploration and mapping," vol. 94, no. 7, pp. 1325-1339, 2006.

[20] Y. Cao, A. Fukunaga, and A. Khang, "Cooperative mobile robotics: Antecedents and directions," Autonomous Robots, vol. 4, no. 1, pp. 7-27, 1997.

[21] G. Dudek, M. Jenkin, E. Milios, and D. Wilkes, "A taxonomy for multiagent robotics," Autonomous Robots, vol. 3, no. 4, pp. 375-397, 1996.

[22] W. Wu and F. Zhang, "A switching strategy for robust cooperative exploration," in Proc. of 2010 IEEE Conf. on Decision and Control, 2010, pp. 5493-5498.

[23] — - "Experimental validation of source seeking with a switching strategy," in IEEE Conference on Robotics and Automation, 2011, pp. $3835-3840$. 